\title{
A Weakly Secure Privacy Preserving Mechanism for Crowd Sensing
}

\author{
Chen Pengfei ${ }^{1, a}$, Zhang Shukui ${ }^{1, b}$, Lin ChengKuan ${ }^{1, c}$
}

1 School of Computer Science and Technology, Soochow University, Suzhou, 215006, China

a871744029@qq.com, b'zhangsk@suda.edu.cn, ccklin@suda.edu.cn

Keywords: Crowd sensing; Network coding; Weak security

\begin{abstract}
The popularity of smart sensing devices is greatly convenient for extensive collection of information. The information collected may include providers' private message, like location, personal identity, and so on. For protecting information providers' privacy, we design the information transfer mechanism WSNC (Weak Security Network Coding) integrated into routing policy. In this mechanism, we design the MWH (Maximizes Wi-Fi Hot), which improve reliability of data transmission. On the basis, we propose privacy protection algorithms based on WSNC which can provide privacy protection. The simulation shows that the privacy protection mechanism based on WSNC not only provides privacy protection also enhances the message delivery ratio.
\end{abstract}

\section{Introduction}

With the development of mobile communication and perceive technology, many innovative applications and services are emerging [1, 2]. They enlarge the dimensions and change the method that people sense the world. Also they open up the new areas of mobile internet, which is called MCS (Mobile Crowd Sensing) [3, 4]. The model of MCS provides a new way for people to participate sensing process while mobile terminals carry out sensing tasks.

We transmit the data that we collected to sensing devices it encounter via Bluetooth technology. Then these sensing devices will send the data they stored to server via Wi-Fi when they move into the area where is the Wi-Fi coverage, which will avoid consuming 3G/4G data flow. The popularization of Wi-Fi provides an extremely good condition to transfer information efficiently. Based on this, we design MWH mobility model. We select the path which passes most Wi-Fi hot to the destination depending on threshold value of the mobility model.

Those people represented by Tracey Ho [5, 6] proposed randomly linear network coding based network coding. When using randomly linear network coding, nodes can select coefficient randomly in the finite fields to code received or stored information and then forward it.

Data transmission between sensing devices informs the Mobile Opportunistic Networks (MONs) [7]. We add WSNC into existing routing protocol [8] and propose the privacy protection mechanism in MONs. Once received information, the sensor node will encode it and then forward it to adjacent nodes. At the same time, the original data achieving the purpose of privacy protection. When receives enough information, server can decode the original information.

\section{Related Work}

Recently, many scholars have done a lot of works in MCS. In [9], authors proposed an efficient information diffusion protocol for sensor data collection via an opportunistic network and this protocol could minimize the information delivery delay. The privacy protection problem in the process of crowd sensing attracts a large researchers attentions. In the paper [10], the authors proposed TAPS to improve the quality of the collected sensor readings by optimizing the participants' selection in proximity of a particular location of interest while still protecting their location privacy. There are some researchers focus on adding noise on the participants' sensor reading to provide privacy, the authors [11] designed PESP and ALPS. 


\section{Mobility Model}

There exist three types of nodes in the network model: (1) sensor node $V=\left\{v_{i} \mid 1 \leq i \leq n\right\}$; (2) Wi-Fi node $\mathrm{W}=\left\{w_{i} \mid 1 \leq i \leq \mathrm{m}\right\}$; (3) server node $\mathrm{F}=\{\mathrm{f}\}$. Sensor node is responsible for sensing and forwarding data, sensor nodes transfer information with Bluetooth, sensor node will deliver the stored data to server node by Wi-Fi once meet Wi-Fi.

The length of shortest path between two point $A$ and $B$ with Map based Dijkstra algorithm in the map denoted as $D(A, B)$, the current location of people is $L_{s}$, the destination is $L_{e}, D_{0}=D\left(L_{s}, L_{e}\right)$. The total path length from $L_{s}$ to $L_{e}$ denoted as $L$. If the path pass $w_{i}$, we set $P\left(w_{i}\right)=1$, otherwise $P\left(w_{i}\right)=0$. The idea of MWH is that when $L-D_{0} \leq \beta$, maximize $\sum_{i=1}^{\delta} P\left(w_{i}\right), \beta$ is a threshold. We use backtracking method to solve this problem, and this problem could turn solve the ordered subsets of $W$. We set $\left(x_{1}, \cdots, x_{\mathrm{n}}\right)$ is an answer, $x_{i}$ means $P\left(w_{i}\right)=1$. Then, we have the follow equation Eq. 1:

$$
L=D\left(L_{s}, w_{x_{1}}\right)+\sum_{i=1}^{n-1} D\left(w_{x_{i}}, w_{x_{i}+1}\right)+D\left(w_{x_{n}}, L_{e}\right) .
$$

When we choose $\left(x_{1}, \cdots \cdots, x_{\mathrm{k}-1}\right)$, the qualification which we choose $x_{k}$ is $x_{k}$ is not equal from $x_{1}$ to $x_{\mathrm{k}-1}$ and $D\left(L_{s}, w_{x_{1}}\right)+\sum_{i=1}^{k-1} D\left(w_{x_{i}}, w_{x_{i}+1}\right)+D\left(w_{x_{n}}, L_{e}\right)-D_{0} \leq \beta$.

\section{Weak Security Network Coding}

A. Weak Security Network Coding Algorithm

The analysis of this section is based on the following information model:

Definition 1 (information receiving model) Node $u \in V$, information $m=$ $\left(\alpha_{1}, \alpha_{2}, \mathrm{~L}, \alpha_{n}\right)\left(x_{1}, x_{2}, \mathrm{~L}, x_{n}\right)^{T}$ arrive $u$, information $m$ can be sensed by node $u$ or relayed by other sensor node. $X=\left(x_{1}, x_{2}, \mathrm{~L}, x_{n}\right)^{T},\left(\alpha_{1}, \alpha_{2}, \mathrm{~L}, \alpha_{n}\right)$ is code vector, note as $A_{0}, m=A_{0} X$. The information packet node $u$ stored which is the same generation as $\mathrm{m}$ is $M$, line $i$ is the $i$-th information packet, denoted as $M_{i}, A$ is $M$ coefficient matrix, $M=A X$. Now connect $A$ with $A_{0}$ as a new matrix, denoted as $A_{\triangle}=\left(A_{0}, A\right)^{\mathrm{T}}$. We design a cache strategy that node $u$ will discard $m$ when $\operatorname{Rank}\left(A_{\triangle}\right) \neq \mathrm{t}+1$.

$M$ will be divided into two set $C$ and $D$, the $i$-th information in set $C$ denoted as $C_{\mathrm{i}}$, the number of messages in set $C$ denoted as $|C|$ and the number of messages in set $D$ denoted as $|D|$. $C$ stores encoded information of $M$, and uncoded information of $M$ will be put in $D$. If $M=\varnothing$, message $m$ will be stored in $u$. When $M \neq \varnothing$, two situations will be discussed:

(1) Information $m$ is sensed by node $u$. if $C \neq \varnothing$, put message $m, D$ and $C_{\mathrm{i}}$ into new set $H$ every time, then carry on random linear network coding with encode function $\operatorname{Code}(H)$, the number of elements in $C$ is $|C|$, then get $|C|$ new information by $|C|$ times encoding. If $C=\varnothing, D$ and $m$ will be merged into $H$ and get $|D|$ messages by $|D|$ times coding with encoding function $\operatorname{Code}(H)$.

(2) Information $m$ is relayed by other sensor nodes. If $D \neq \varnothing, D$ and $m$ will be merged into $H$ and get $|D|$ messages by coding $|D|$ times with encoding function $\operatorname{Code}(H)$. If $C \neq \varnothing$, put $m$ and $C_{\mathrm{i}}$ into new set $H$ and then carry on random linear network coding with encoding function $\operatorname{Code}(H)$, the number of elements in $C$ is $|C|$, then get $|C|$ new information by $|C|$ times coding.

Finally, deleting the original information in $M$ and saving the information encoded in node $u$.

Coding function Code $(H)$, a linear combination of information by random linear network coding in the finite field, all operations are completed in $\mathbb{F}_{q}$. If $H=\left(\sum_{j=1}^{n} g_{j}{ }^{1} x_{j}, \sum_{j=1}^{n} g_{j}{ }^{2} x_{j}, \ldots, \sum_{j=1}^{n} g_{j}{ }_{j} x_{j}\right)^{\mathrm{T}}$, when conduct random linear network coding, select $l$ coefficients $k_{1}, k_{2}, \mathrm{~L}, k_{l}$ from the finite field randomly, after encoding, get information packets $Z=\sum_{i=1}^{l}\left(k_{i} \sum_{j=1}^{n} g_{j}{ }_{j} x_{j}\right)$.

B. Decode Algorithm

In the network, only server node $f$ can decode, information can recover the original data by decoding. We can use Gauss elimination in decoding algorithm, for the same generation packets 
$X=\left(x_{1}, x_{2}, \mathrm{~L}, x_{n}\right)$, if data packets serve node $\mathrm{f}$ received is $Z, Z=\left(\sum_{j=1}^{n} \alpha_{j}{ }_{j} x_{j}, \sum_{j=1}^{n} \alpha_{j}{ }^{2} x_{j}, \ldots, \sum_{j=1}^{n} \alpha_{j}{ }^{s} x_{j}\right)^{\mathrm{T}}$, the coefficient matrix is denoted by $A, Z=A X$. When $f$ can recover part of the source code package by received information without waiting for that $\operatorname{Rank}(A)=n$.

Supposing that the received information $m$ has been processed by cache strategy, and just consider the information packets in $f$ as the same generation of $m$. Firstly, add $m$, the information decoded by $f$ and message not decoded in $f$ into $Z$, and mark the information decoded by $f$. $Z=A X$, and operate Gauss elimination on $Z, Z^{\prime}=A^{\prime} X$. For each line vector $A_{\mathrm{i}}^{\prime}$ in $A^{\prime}$, if $A_{\mathrm{i} j}^{\prime}=1$ and $\sum_{k=1, k \neq j}^{n} A_{\mathrm{i} j}^{\prime}=0$ when $x_{j}$ is not solved, then $x_{j}$ can be solved, $x_{j}=Z^{\prime}{ }_{i}$.

\section{Simulations And Performance Analysis}

In this section, we use the ONE simulation platform to simulate and analyze MWH mobility model and WSNC algorithm. We set Bluetooth transmit speed is $250 \mathrm{Kbps}$ and transmission range is $10 \mathrm{~m}$, Message created interval is 15 25s, movement speed of sensor nodes is $1.5 \sim 30 \mathrm{~m} / \mathrm{s}$.

A. Compared the network performance under different number of sensor nodes.

We set the packet size is $500 \mathrm{~kb}$, the number of sensor nodes is 10 times of number of Wi-Fi nodes, $\beta$ is $1000 \mathrm{~m}$. With the increase of number of nodes, the more frequent exchange of message between nodes, so that the message is more likely to be forwarded to more relay nodes, reduces the message delivery delay. From Fig. 1 and Fig.2, we can obtain that the delivery ratio increases with the number of sensor nodes and Wi-Fi nodes. WSNC has higher deliver rate and average delivery delay.

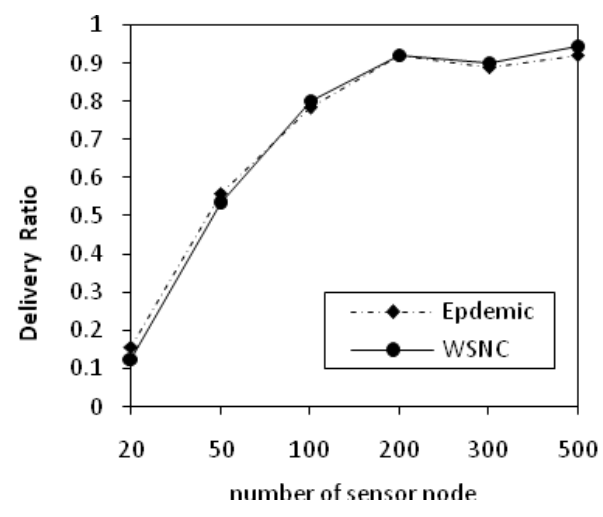

Fig. 1. Delivery ratio under different number of nodes.

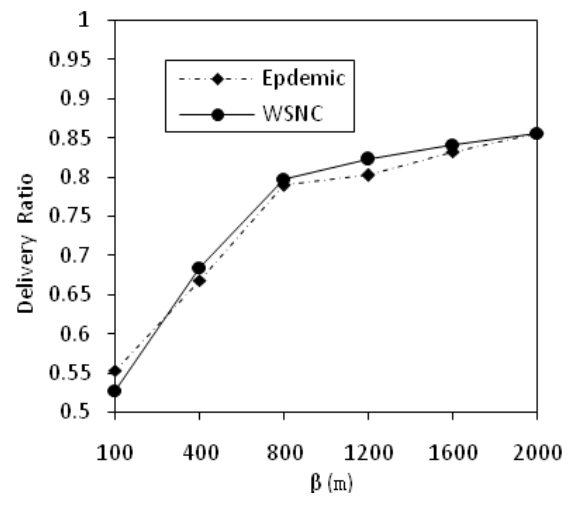

Fig. 3. Delivery ratio under different $\beta$.

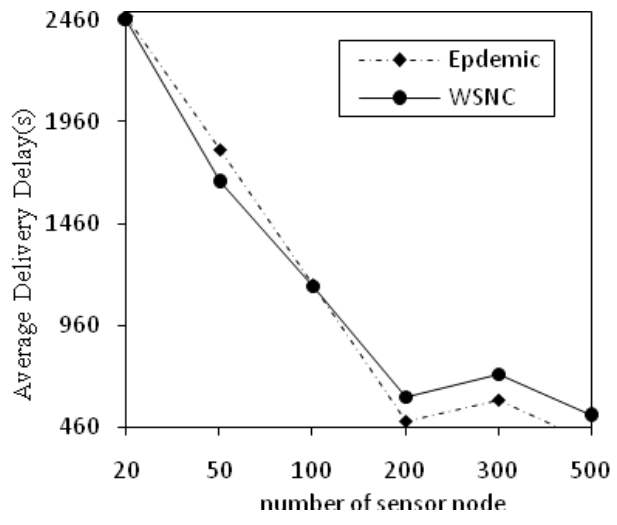

Fig. 2. Delivery delay under different number of nodes

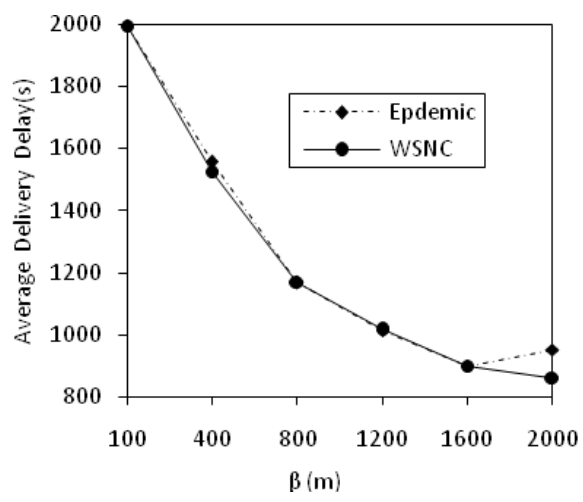

Fig. 4. Delivery delay under different $\beta$

B. Compared network performance under different $\beta$ value in MWH mobility model 
We set the packet size of $500 \mathrm{~kb}$, the number of sensor nodes is 100 , and the number of Wi-Fi nodes is 20 . When the $\beta$ value is small, the path pass Wi-Fi relative lower, he delivery ratio and the average delivery delay is larger due to message delivered to server only by Wi-Fi. When the $\beta$ value is larger, the delivery ratio increases, the average delivery delay is small, the impact of changes in $\beta$ value becomes smaller, and then the delivery ratio, average delivery delay variation rate becomes smaller. From Fig.3 and Fig.4 can be seen, WSNC has higher deliver rate. We can conclude that the delivery ratio of $\mathrm{MWH}$ increased significantly meanwhile the average delivery delay reduced significantly.

\section{Summary}

In this paper, we proposed an information transfer mechanism that WSNC is integrated into routing policy. MWH mobility model enhances the quality and efficiency of data transmission greatly. The original information will be hidden for privacy protection of the information when forwarding the collected information to neighbor node by WSNC algorithm.

\section{Acknowledgements}

This work is supported by National Natural Science Foundation of China under Grants No.61070169, 61201212 and Natural Science Foundation of Jiangsu Province under Grant No. BK2011376.

\section{References}

[1] N.D. Lane, E. Miluzzo, L. Hong, D. Peebles, T. Choudhury, and A.T. Campbell, “A survey of mobile phone sensing,” IEEE Communications Magazine, vol. 48, no. 9, pp. 140-150, 2010.

[2] H. Ma, D. Zhao, and P. Yuan, “Opportunities in mobile crowd sensing,” IEEE Communications Magazine, vol. 52, no. 8, pp. 29-35, 2014.

[3] R. K. Ganti, Y. Fan, and L. Hui, "Mobile crowd sensing: current state and future challenges," IEEE Communications Magazine, vol. 49, no. 11, pp. 32-39, 2011.

[4] B. Guo, Z. Yu, "From Participatory Sensing to Mobile Crowd Sensing,” IEEE International Conference on Pervasive Computing and Communications Workshops, pp. 593-598, 2014.

[5] R. Ahlswede, C. Ning, S. Y. R. Li, and R. W. Yeung, "Network information flow" IEEE Transactions on Information Theory, vol. 46, no. 4, pp. 1204-1216, 2000.

[6] H. Tracey, M. Medard, R. Koetter, and D. R. Karger, “A random linear network coding approach to multicast,” IEEE Transactions on Information Theory, vol. 52, no. 10, pp. 4413-4430, 2006.

[7] L. Pelusi, A. Passarella, “Opportunistic networking: data forwarding in disconnected mobile ad hoc networks,” IEEE Communications Magazine, vol. 44, no. 11, pp. 134-141, 2006.

[8] A. Vahdat, and D. Becker, “Epidemic Routing for Partially-Connected Ad Hoc Networks,” Duke University, Technical report CS-200006, 2000.

[9] T. Higuchi, H. Yamaguchi, "A neighbor collaboration mechanism for mobile crowd sensing in opportunistic networks,” IEEE International Conf. on IEEE Communications, pp. 42-47, 2014.

[10] L. Kazemi, C. Shahabi. TAPAS: trustworthy privacy-aware participatory sensing, Knowl. Inf. Syst, vol. 37, no. 1, pp. 105-128, 2013.

[11] F. Zhang, L. He, W. He. Data perturbation with state-dependent noise for participatory sensing. Proceedings of the 31st IEEE Conference on Computer Communications, pp. 2246-2254, 2012. 